\title{
Negative Ion Photoelectron Spectroscopy of $\mathrm{SiN}^{-\dagger}$
}

\author{
Giovanni Meloni, Sean M. Sheehan, Michael J. Ferguson, and Daniel M. Neumark* \\ Department of Chemistry, University of California, and Chemical Sciences Division, \\ Lawrence Berkeley National Laboratory, Berkeley, California 94720
}

Received: May 15, 2004; In Final Form: June 24, 2004

\begin{abstract}
Negative ion photoelectron spectra of $\mathrm{SiN}^{-}$have been recorded using the $355 \mathrm{~nm}(3.493 \mathrm{eV})$ and $266 \mathrm{~nm}$ $(4.661 \mathrm{eV})$ photodetachment wavelengths. The spectra exhibit resolved vibrational features corresponding to transitions to the $\mathrm{X}^{2} \Sigma^{+}$and $\mathrm{A}{ }^{2} \Pi$ states of SiN. Franck-Condon analyses yield the first experimental spectroscopic parameters, $r_{\mathrm{e}}$ and $\omega_{\mathrm{e}}$, for the anion ground state, $\mathrm{X}^{1} \Sigma^{+}$. We also determined the first experimental adiabatic electron affinity as $2.949 \pm 0.008 \mathrm{eV}$. The anion dissociation energy $D_{0}\left(\mathrm{SiN}^{-}\right)$is then obtained from the electron affinities of $\mathrm{Si}$ and $\mathrm{SiN}$ and the dissociation energy of the neutral.
\end{abstract}

\section{Introduction}

Silicon nitride $\left(\mathrm{Si}_{3} \mathrm{~N}_{4}\right)$ is a material of considerable interest because of its remarkable properties such as strength, hardness, chemical inertness, good resistance to wear and corrosion, high thermal stability, and good dielectric properties. ${ }^{1}$ This combination of properties makes $\mathrm{Si}_{3} \mathrm{~N}_{4}$ a very attractive highperformance engineering material. For instance, its low density, $40 \%$ less than those of high-temperature superalloys, may offer components with lower weight and inertia, which would improve the performance of engines. Another important characteristic is the universal abundance and consequent low cost of the elements silicon and nitrogen in contrast with the short supply and increasing costs of metals for high-temperature alloys such as chromium, cobalt, nickel, and tungsten. ${ }^{1}$

Numerous studies of silicon nitride have been performed ${ }^{2-7}$ to characterize many properties of the bulk, but only a few spectroscopic investigations have been carried out to study the electronic structure of its molecular subunits. In this paper we present an experimental study on the low-lying electronic states of $\mathrm{SiN}^{-}$and $\mathrm{SiN}$ using negative ion photoelectron spectroscopy (PES). A major advantage of anion PES over optical spectroscopy studies is that the transition selection rules are less restrictive. In principle all one-electron transitions are allowed; for instance, this enables us to access both doublet and quartet states from the photodetachment of a triplet electronic state. Moreover, mass selection allows us to unambiguously isolate the ion of interest. Anion PES has been successfully applied in our group to gain information on electron affinities, term energies, and vibrational frequencies for many diatomics such as $\mathrm{CN},{ }^{8} \mathrm{BN},{ }^{9} \mathrm{AlP}, \mathrm{GaP}$, InP, and GaAs. ${ }^{10}$

Emission spectra assigned to the $\mathrm{SiN}$ radical have been observed by many investigators, starting with experiments by Jevons ${ }^{11}$ in 1913 and Mulliken ${ }^{12}$ in 1925 . The $B^{2} \Sigma^{+}-X^{2} \Sigma^{+}$ transition, with its band origin at $24299 \mathrm{~cm}^{-1}$, has been well understood for some time. However, characterization of the lower lying $A^{2} \Pi$ state proved more difficult. Bredohl et al. ${ }^{13}$ explored the emission spectrum of $\mathrm{SiN}$ and gave an approximate term energy $\left(T_{\mathrm{e}}\right)$ of $8000 \mathrm{~cm}^{-1}$ for the $A^{2} \Pi_{\Omega}$ electronic state.

\footnotetext{
† Part of the special issue "Tomas Baer Festschrift".

* To whom correspondence should be addressed at the University of California.
}

A much smaller value, $994 \mathrm{~cm}^{-1}$, was obtained by analysis of $B^{2} \Sigma^{+}-A^{2} \Pi$ emission by Foster ${ }^{14}$ and $A^{2} \Pi-X^{2} \Sigma^{+}$absorption by Yamada and Hirota. ${ }^{15}$ The value of $T_{\mathrm{e}}$ from the absorption experiment relied on the assignment of a band around $2000 \mathrm{~cm}^{-1}$ to the $1-0$ vibronic transition between the $A$ and $X$ states. In a subsequent experiment by Yamada et al., ${ }^{16}$ this band was reassigned to the $0-0$ transition on the basis of isotope shifts, yielding $T_{\mathrm{e}}=2032.4 \mathrm{~cm}^{-1}$ for the $A$ state. On the basis of this assignment, Foster ${ }^{17}$ performed a global fit of term energies and vibrational frequencies of the ground and several excited states of $\mathrm{SiN}$.

Saito et al. ${ }^{18}$ detected the microwave absorption spectrum of $\mathrm{SiN}$, from which an equilibrium distance $\left(r_{\mathrm{e}}\right)$ of $1.5721 \AA$ for the ground electronic state is obtained. Naulin et al., ${ }^{19}$ through a pulsed crossed molecular beam study of the $\mathrm{Si}+\mathrm{N}_{2} \mathrm{O} \rightarrow$ $\mathrm{SiN}+\mathrm{NO}$ reaction, determined the bond dissociation energy of $4.68 \mathrm{eV}$ for $\mathrm{SiN}$, which compares well with the Gaussian-2 theory value of $4.58 \mathrm{eV}$ by Curtiss et al. ${ }^{20}$

Several calculations have been performed on the ground and excited electronic states of $\mathrm{SiN}^{21-27}$ In particular, a detailed investigation on $\mathrm{SiN}$ was carried out by Cai et al., ${ }^{23}$ who calculated the spectroscopic molecular parameters $\left(r_{\mathrm{e}}, \omega_{\mathrm{e}}, \omega_{\mathrm{e}} x_{\mathrm{e}}\right.$, $\omega_{\mathrm{e}} y_{\mathrm{e}}$, rotational constants $\left(B_{\mathrm{e}}\right)$, centrifugal distortion constants $\left(D_{\mathrm{e}}\right)$, dipole moments $\left(\mu_{0}\right), T_{\mathrm{e}}$, and dissociation energies $\left.\left(D_{0}\right)\right)$ for 11 bound doublet states and 10 bound quartet states of SiN. They used the internally contracted multireference configuration interaction (CMRCI) level of theory with Dunning's correlationconsistent polarization valence quadruple- $\zeta$ (cc-pVQZ) basis set to study the potential energy curves for the low-lying electronic states.

Less is known about the anion $\mathrm{SiN}^{-}$. There is no experimental determination of the electron affinity (EA). Peterson and Woods $^{21}$ calculated the EA of SiN as $3.33 \mathrm{eV}$ using the MøllerPlesset perturbation theory with single, double, and quadruple substations (MP4SDQ). Kalcher ${ }^{25}$ employed the complete active space in conjunction with the averaged coupled pair functional approach (CAS-ACPF) with the cc-pVQZ basis set to investigate trends in ground and excited electronic states of group 14, 15, and 16 mixed diatomic anions. He computed an EA of $2.862 \mathrm{eV}$ and also calculated a bound excited ${ }^{3} \Sigma^{+}$state of $\mathrm{SiN}^{-}$ lying only $0.341 \mathrm{eV}$ below the $\mathrm{SiN}^{2} \Sigma^{+}$ground state. $\mathrm{SiN}$ and $\mathrm{SiN}^{-}$are isovalent with $\mathrm{CN}$ and $\mathrm{CN}^{-}$, and have the same 
ordering of electronic states. However, no calculations have found $\mathrm{CN}^{-}$to have any stable excited electronic states.

In this investigation, we used negative ion PES to examine the electronic structure of the ground and low-lying excited electronic states of $\mathrm{SiN}$. The PE spectrum provides a straightforward confirmation of the $\mathrm{A}^{2} \Pi$ state term value. We obtain the first experimental value of the $\mathrm{EA}(\mathrm{SiN})$, and using this result, we derive the $\mathrm{SiN}^{-}$dissociation energy. With the aid of a Franck-Condon (FC) simulation we determine the equilibrium bond distance and vibrational frequency of the ground electronic state of the anion $\mathrm{SiN}^{-}$. We also tentatively assigned a minor peak in the PE spectra to a transition between the first excited state of $\mathrm{SiN}^{-}\left({ }^{3} \Sigma^{+}\right)$and the ${ }^{4} \Pi_{\Omega}$ state, on the basis of the calculated relative energetics of these states and electronic configuration considerations.

\section{Experiment}

Details of the anion time-of-flight (TOF) PES apparatus used in this study have been described previously. ${ }^{28,29} \mathrm{SiN}$ anions are generated using a pulsed laser ablation molecular beam source. The second harmonic output (532 nm, 10-15 mJ/pulse) of a pulsed $\mathrm{Nd}$ :YAG laser operated at $20 \mathrm{~Hz}$ is focused through a $35 \mathrm{~cm}$ focal lens onto a rotating and translating pure silicon nitride disk (Crystallod Inc.). The resulting plasma is entrained into a pulsed supersonic beam of Ar. The gas pulse then enters a linear reflectron TOF mass spectrometer, where the negative ions are extracted perpendicular to their flow direction by a pulsed electric field and accelerated to $2.5 \mathrm{keV}$ beam energy. The mass resolution $m / \Delta m$ is approximately 2000 .

The chosen ion is then selectively photodetached by a pulsed fixed-frequency Nd:YAG laser using the third $(355 \mathrm{~nm}$ or 3.493 $\mathrm{eV}$ ) and fourth (266 nm or $4.661 \mathrm{eV})$ harmonics. Photoelectrons are collected perpendicularly to the ion and laser beam plane, and the electron kinetic energy (eKE) distribution is determined by TOF analysis in a $1 \mathrm{~m}$ field-free flight tube. We calibrate the eKE scale using the PE spectra of $\mathrm{Cl}^{-}, \mathrm{Br}^{-}$, and $\mathrm{I}^{-}$at 266 $\mathrm{nm}$ photon wavelength, and $\mathrm{O}_{2}^{-}$at $355 \mathrm{~nm}$ photon wavelength. The energy resolution is $8-10 \mathrm{meV}$ at $0.65 \mathrm{eV}$ eKE and degrades as $(\mathrm{eKE})^{3 / 2}$ at higher eKE.

All PE spectra presented here are plotted as a function of the electron binding energy (eBE) defined as

$$
\mathrm{eBE}=h v-\mathrm{eKE}=\mathrm{EA}+E^{(0)}-E^{(-)}
$$

where $h v=3.493$ or $4.661 \mathrm{eV}$ is the photodetachment energy, $E^{(0)}$ is the internal energy of the neutral, and $E^{(-)}$is the internal energy of the anion.

This anion TOF PES apparatus also yields the angular distribution of the detached photoelectrons. The PE spectra were taken at two laser polarization angles, $0^{\circ}$ and $90^{\circ}$, with respect to the direction of the electron detection. This angle can be varied using a half-wave plate. The angular distribution of the photoelectrons is given by ${ }^{30}$

$$
\frac{\mathrm{d} \sigma}{\mathrm{d} \Omega}=\frac{\sigma_{\text {total }}}{4 \pi}\left[1+\frac{\beta}{2}\left(3 \cos ^{2} \theta-1\right)\right]
$$

where $\theta$ is the angle between the laser electric field and the direction of electron detection, $\sigma_{\text {total }}$ is the total photodetachment cross section, and $\beta$ is the anisotropy parameter, which varies from -1 to +2 . Each neutral $\leftarrow$ anion electronic transition has a characteristic $\beta$ that can be used to distinguish peaks of overlapping electronic transitions, and can be calculated using

$$
\beta=\frac{I_{0^{\circ}}-I_{90^{\circ}}}{1 / 2 I_{0^{\circ}}+I_{90^{\circ}}}
$$

where $I_{0^{\circ}}$ and $I_{90^{\circ}}$ are the intensities of the peak taken at $\theta=0^{\circ}$ and $90^{\circ}$.

\section{Results}

The 355 and $266 \mathrm{~nm}$ photoelectron spectra of $\mathrm{SiN}^{-}$taken at laser polarization angles $\theta$ of $90^{\circ}$ and $0^{\circ}$ are displayed in Figure 1. The PE signal is plotted in terms of eBE. Anisotropy parameters $\beta$ were determined for selected bands and are indicated by the solid black diamonds on the top panel of Figure 1. Peak positions are listed in Table 1 .

The $355 \mathrm{~nm}$ spectrum at $90^{\circ}$ shows four main peaks labeled $\mathrm{X}_{0,1}$ and $\mathrm{A}_{0,1}$. The two most intense peaks, $\mathrm{X}_{0}$ and $\mathrm{A}_{0}$, have very different anisotropy parameters of 0.7 and -0.5 , respectively, and appear to be vibrational origins of two electronic transitions, while peaks $X_{1}$ and $A_{1}$ are transitions to vibrationally excited levels within the two electronic bands. The $266 \mathrm{~nm}$ spectra reveal many of the same peaks seen in the $355 \mathrm{~nm}$ spectra but with lower resolution because of higher electron kinetic energy. Two additional peaks, $\mathrm{A}_{2}$ and $\mathrm{A}_{3}$, are observed. Peaks $\mathrm{A}_{0}-\mathrm{A}_{3}$ are equally spaced and have the same $\beta$ parameter, and thus appear to represent a vibrational progression in a single electronic band.

In the $355 \mathrm{~nm}$ spectrum, peaks $\mathrm{X}_{0}$ and $\mathrm{A}_{0}$ are spaced by 2010 $\mathrm{cm}^{-1}$, very close to the accepted splitting between the $X^{2} \Sigma^{+}$ and $A{ }^{2} \Pi$ states of $\mathrm{SiN} .{ }^{16,17}$ Moreover, peak $\mathrm{A}_{0}$ appears to comprise two partially resolved peaks split by $90 \mathrm{~cm}^{-1}$, in good agreement with the spin-orbit coupling constant of -89.097 $\mathrm{cm}^{-115}$ for the $\mathrm{SiN} A^{2} \Pi$ state. The $\mathrm{SiN}^{-}$anion is predicted to have an $X^{1} \Sigma^{+}$ground state with no excited states lying nearby, so we assign peaks $\mathrm{X}_{0}$ and $\mathrm{A}_{0}$ to the vibrational origins of the $X^{2} \Sigma^{+} \leftarrow X^{1} \Sigma^{+}$and $A^{2} \Pi \leftarrow X^{1} \Sigma^{+}$transitions. Since peak $\mathrm{X}_{0}$ occurs at $\mathrm{eBE}=2.95 \mathrm{eV}$, this is the approximate electron affinity of SiN. Peaks $\mathrm{X}_{0}$ and $\mathrm{X}_{1}$ are spaced by $1160 \mathrm{~cm}^{-1}$, while $A_{0}$ and $A_{1}$ are spaced by $1060 \mathrm{~cm}^{-1}$; these should be the vibrational fundamental frequencies of the $X^{2} \Sigma^{+}$and $A^{2} \Pi$ states, respectively, and are indeed very close to the literature values. ${ }^{17}$

There are two smaller peaks in the spectra at $\mathrm{eBE}=2.81$ and $3.04 \mathrm{eV}$. Each lies at $1100 \mathrm{~cm}^{-1}$ lower eBE from one of the vibronic origin peaks, $\mathrm{X}_{0}$ or $\mathrm{A}_{0}$, and has the same anisotropy parameter at that origin peak. These features are assigned to anion vibrational hot bands, yielding an anion vibrational frequency of approximately $1100 \mathrm{~cm}^{-1}$, and each is labeled $\mathrm{HB}$ in Figure 1. There appears to be an additional small feature, labeled a (see Figure 2), at slightly lower eBE than the hot band at $3.04 \mathrm{eV}$. At both wavelengths, this feature has the same anisotropy parameter as the peaks $\mathrm{A}_{n}$. Its possible assignment is discussed below.

\section{Analysis and Discussion}

To extract additional and more precise information on the anion and neutral, the PE spectrum was simulated within the Franck-Condon approximation. Within each electronic band, the vibrational transitions have intensities proportional to their FC factors, $\left|\left\langle Y_{v^{\prime}} \mid \Psi_{v}\right\rangle\right|^{2}$, where $\Psi_{v^{\prime}}$ and $\Psi_{v}$ are neutral and anion Morse oscillator wave functions, respectively. An anion vibrational temperature of $1000 \mathrm{~K}$ was assumed in the simulations. 

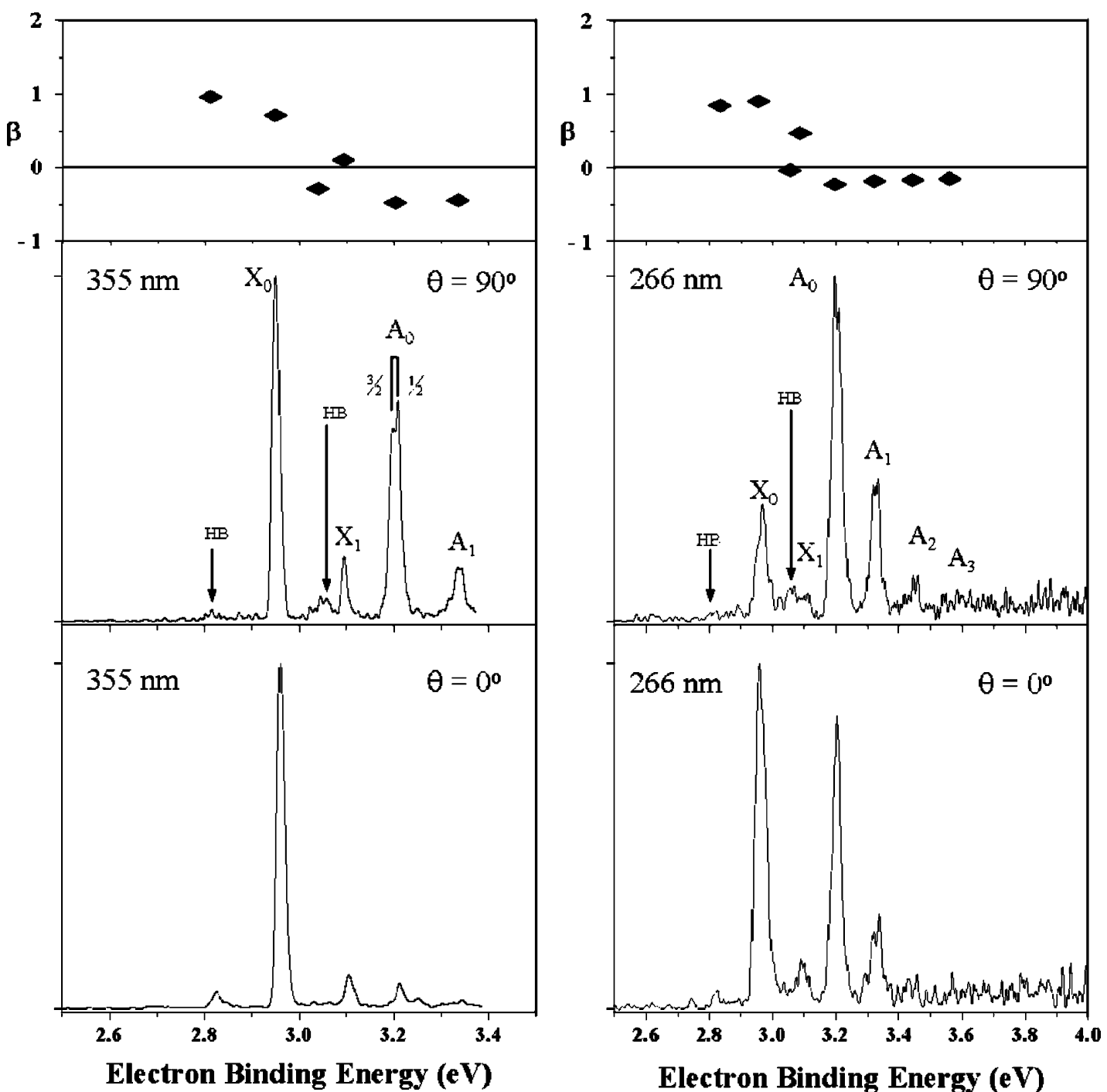

Figure 1. Anion photoelectron spectra of $\mathrm{SiN}^{-}$taken at 355 and $266 \mathrm{~nm}$ with the laser polarization angle $\theta=90^{\circ}$ and $0^{\circ}$. The values of the asymmetry parameters $\beta$ are indicated by the solid black diamonds above the PE spectra for selected bands.

TABLE 1: Franck-Condon Simulation Optimized Parameters, $r_{\mathrm{e}}(\AA), \omega_{\mathrm{e}}\left(\mathrm{cm}^{-1}\right)$, and $T_{\mathrm{e}}\left(\mathrm{cm}^{-1}\right)^{a}$

\begin{tabular}{cccccccc}
\hline & & band & & & & $\omega_{\mathrm{e}}$ & $T_{\mathrm{e}}$ \\
species & band & origin & state & \multicolumn{1}{c}{$r_{\mathrm{e}}$} & $1130 \pm 20$ & 0.000 & 1000 \\
$\mathrm{SiN}^{-}$ & & & $X^{1} \Sigma^{+}$ & $1.604 \pm 0.005$ & $1151^{b}$ & 0.000 \\
$\mathrm{SiN}^{2}$ & $\mathrm{X}$ & 2.949 & $X^{2} \Sigma^{+}$ & $1.572^{b}$ & $1032^{d}$ & $(2008 \pm 65)+1 / 2 A_{\mathrm{e}}{ }^{e}$
\end{tabular}

${ }^{a}$ The band origin is in electronvolts. ${ }^{b}$ Reference $16 .{ }^{c}$ Reference $15 .{ }^{d}$ Reference $14 .{ }^{e} A_{\mathrm{e}}$ was taken from Yamada and Hirota ${ }^{15}$ as $89.097 \mathrm{~cm}^{-1}$.

This value was chosen to reproduce the hot band intensities in the PE spectra, and it does not have any effect on the determination of the energetics and molecular parameters. Such a high vibrational temperature is not uncommon in laser ablation experiments in which conditions are optimized for the production of diatomic anions, as was seen in our previous study of $\mathrm{C}_{2}-31$

For each anion and/or neutral state, the parameters that determine these wave functions are $r_{\mathrm{e}}$, the equilibrium bond length, $\omega_{\mathrm{e}}$, the harmonic vibrational frequency, and $\omega_{\mathrm{e}} x_{\mathrm{e}}$, the anharmonicity. All of these parameters are known for the neutral $\mathrm{X}$ and $\mathrm{A}$ states, ${ }^{16-18}$ and were fixed at those values (see Table 1) in the simulations. The experimental spin-orbit coupling constant ${ }^{15} A_{\mathrm{e}}=-89.097 \mathrm{~cm}^{-1}$ was used for the $\mathrm{A}^{2} \Pi$ state, as was the experimental value of $2032 \mathrm{~cm}^{-1}$ for the term value of this state. Thus, only the anion parameters needed to be varied: the bond length, vibrational frequency, and $\mathrm{EA}(\mathrm{SiN})$.
Our assignment described in section 3 indicated only a single hot band transition associated with each of the two photodetachment bands, so that the anion anharmonicity cannot be extracted from our simulations. We thus estimated $\omega_{\mathrm{e}} x_{\mathrm{e}}$ for $\mathrm{SiN}^{-}$ to be $4.3 \mathrm{~cm}^{-1}$ by scaling it to that of the neutral using the Morse function formula:

$$
\frac{\omega_{\mathrm{e}} x_{\mathrm{e}}^{(-)}}{\omega_{\mathrm{e}} x_{\mathrm{e}}^{(0)}}=\frac{\left(\omega_{\mathrm{e}}^{(-)}\right)^{2}}{\left(\omega_{\mathrm{e}}^{(0)}\right)^{2}} \frac{D_{\mathrm{e}}^{(0)}}{D_{\mathrm{e}}^{(-)}}
$$

where $D_{\mathrm{e}}^{(0)}$ and $D_{\mathrm{e}}^{(-)}$are the neutral and anion dissociation energies (the determination of $D_{\mathrm{e}}^{(-)}$is discussed below). The simulated PE spectra are shown in Figure 2, and best fit anion parameters are displayed in Table 1.

The PE spectrum yields the first experimental determination of the $\mathrm{SiN}$ adiabatic electron affinity, $2.949 \pm 0.008 \mathrm{eV}$, slightly 


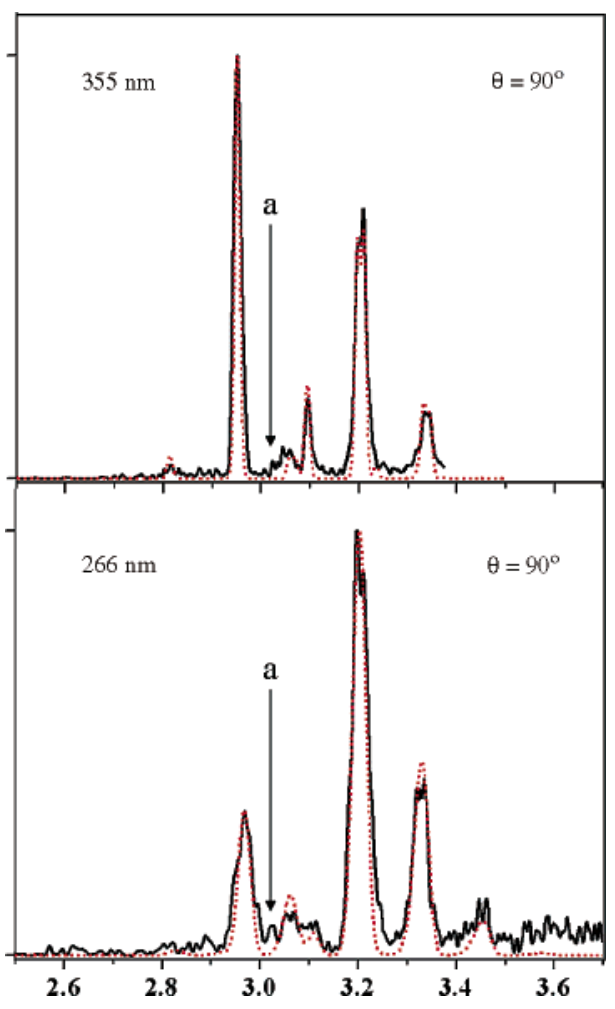

Figure 2. Anion photoelectron spectra of $\mathrm{SiN}^{-}$taken at $355 \mathrm{~nm}$ with $\theta=0^{\circ}$ and $266 \mathrm{~nm}$ with $\theta=90^{\circ}$. The FC-simulated (dotted) PE spectra are superimposed on the experimental ones.

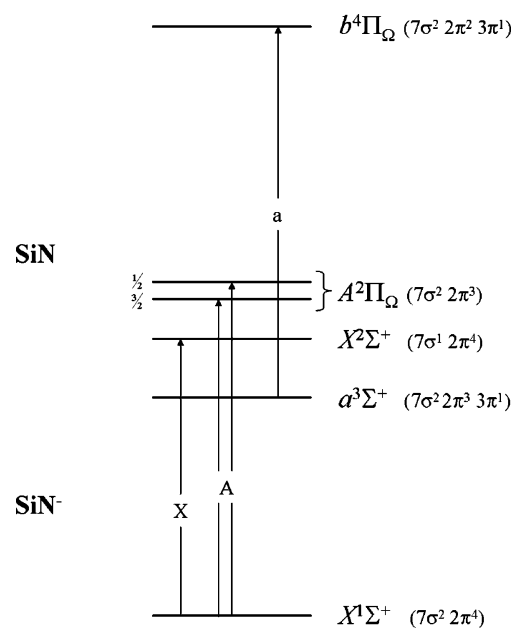

Figure 3. Energy level diagram for $\mathrm{SiN}^{-} / \mathrm{SiN}$ as derived from ab initio, DFT, and experimental investigations.

higher than the value of $2.8632 \mathrm{eV}$ recently calculated by Kalcher. ${ }^{25}$ The resulting energetics for the anion and neutral states, along with the dominant molecular orbital configurations, are shown in Figure 3. Our FC analysis also yields the first experimental determination of the molecular constants for the anion $\mathrm{SiN}^{-}$. In particular, using $r_{\mathrm{e}}=1.572066 \pm 0.000041 \AA^{18}$ for the SiN ground state and $r_{\mathrm{e}}=1.641879 \pm 0.000020 \AA^{16}$ for the $A^{2} \Pi$ state, in combination with the bond changes for the $X^{2} \Sigma^{+}+\mathrm{e}^{-} \leftarrow X^{1} \Sigma^{+}$and $A^{2} \Pi_{\Omega}+\mathrm{e}^{-} \leftarrow X^{1} \Sigma^{+}$electronic transitions determined from the FC simulation, we obtain $r_{\mathrm{e}}\left(\mathrm{SiN}^{-}\right)=1.604 \pm 0.005 \AA$. Note that while one can generally only extract the absolute value of the bond length change $\left|\Delta r_{\mathrm{e}}\right|$ from FC analysis, the observation of transitions to two neutral electronic states with known bond lengths removes any ambiguity in $r_{\mathrm{e}}$ for the anion. Finally, from the FC analysis, we also determined $\omega_{\mathrm{e}}\left(\mathrm{SiN}^{-}\right)=1130 \pm 20 \mathrm{~cm}^{-1}$; this was optimized to reproduce the position of the hot band in the PE spectrum. The molecular parameters obtained from the PE spectrum simulation are also in very good agreement with Kalcher's calculations $\left(r_{\mathrm{e}}=1.6045 \AA\right.$ and $\left.\omega_{\mathrm{e}}=1120 \mathrm{~cm}^{-1}\right){ }^{25}$

The lowest dissociation asymptote of $\mathrm{SiN}^{-}$is $\mathrm{Si}^{-}\left(3 \mathrm{p}^{3},{ }^{4} \mathrm{~S}_{3 / 2}\right)$ $+\mathrm{N}\left(2 \mathrm{p}^{3},{ }^{4} \mathrm{~S}_{3 / 2}\right)$; therefore, from a thermodynamic cycle we can derive the dissociation energy difference between $\mathrm{SiN}^{-}$and SiN employing the relation $D_{0}\left(\mathrm{SiN}^{-}\right)-D_{0}(\mathrm{SiN})=\mathrm{EA}(\mathrm{SiN})$ - EA(Si). Using the EA(SiN) value of $2.949 \mathrm{eV}$ determined in this study together with the $\mathrm{EA}(\mathrm{Si})$ value of $1.3895 \mathrm{eV}$ measured by Blondel et al., ${ }^{32}$ we obtain $D_{0}\left(\mathrm{SiN}^{-}\right)-D_{0}(\mathrm{SiN})=1.560 \pm$ $0.008 \mathrm{eV}$. In addition, using the $D_{0}(\mathrm{SiN})$ value of $4.68 \pm 0.14$ $\mathrm{eV}$ derived by Naulin et al. ${ }^{19}$ from a crossed molecular beam study, we derive the dissociation energy of $\mathrm{SiN}^{-}$as $6.24 \pm 0.14$ $\mathrm{eV}$.

Owing to the relatively small amounts of $\mathrm{SiN}^{-}$produced in the ion source, the PE spectra are not entirely noise-free, even at $355 \mathrm{~nm}$ where there is virtually no background from stray photoelectrons. There are small features in the PE spectra that are not fit by the above simulations. The most reproducible of these is labeled "a" at eBE $=3.04 \mathrm{eV}$ in the $355 \mathrm{~nm}$ PE spectra shown in Figure 2. There are no other neutral states of SiN accessible from the anion ground state in this energy range. ${ }^{17,23}$ This feature may arise from photodetachment of the a ${ }^{3} \Sigma+$ excited state of $\mathrm{SiN}^{-}$, calculated by Kalcher ${ }^{25}$ to lie $0.341 \mathrm{eV}$ below the detachment threshold. The molecular orbital configuration of this state is ...7 $\sigma^{2} 2 \pi^{3} 3 \pi^{1}$. The anisotropy parameter for peak "a" is similar to that for the main progression $\mathrm{A}_{0-n}$, suggesting that the photodetachment transition involves removal of an electron from the same orbital as in the $A^{2} \Pi \leftarrow X^{1} \Sigma^{+}$ transitions, namely, the $2 \pi$ orbital. Detachment of this electron from the $a^{3} \Sigma^{+}$state produces the ${ }^{4} \Pi$ neutral state, as shown in Figure 3. The calculation by Cai et al. ${ }^{23}$ found $T_{\mathrm{e}}=2.83 \mathrm{eV}$ for the $b^{4} \Pi$ state, and using this along with Kalcher's energy yields eBE $=3.17 \mathrm{eV}$ for the $b^{4} \Pi \leftarrow a^{3} \Sigma^{+}$photodetachment transition, remarkably close to peak "a". Unfortunately, we did not observe the $\mathrm{A}^{2} \Pi+\mathrm{e}^{-} \leftarrow \mathrm{a}^{3} \Sigma^{+}$transition that would allow us to determine unequivocally the term energies of the triplet anion and neutral quartet state.

Finally, it is worthwhile to compare the experimental findings for $\mathrm{SiN}^{-} / \mathrm{SiN}$ with those for the isovalent molecules $\mathrm{CN}^{-} / \mathrm{CN}$. The EA of CN, $3.862 \pm 0.004 \mathrm{eV},{ }^{8}$ is considerably higher than $\mathrm{EA}(\mathrm{SiN})$ found here. The dissociation energies of $\mathrm{CN}^{-}, 10.31$ $\mathrm{eV},{ }^{33}$ and $\mathrm{CN}, 7.76 \mathrm{eV},{ }^{33}$ are much higher than $D_{0}\left(\mathrm{SiN}^{-}\right)$and $D_{0}(\mathrm{SiN}) . \mathrm{CN}^{-}$is a closed shell anion with an $X^{1} \Sigma^{+}$ground state, while $\mathrm{CN}$ has an $X^{2} \Sigma^{+}$ground state and low-lying $A^{2} \Pi$ and $B^{2} \Sigma^{+}$states. While the state ordering is the same as in $\mathrm{SiN}, T_{\mathrm{e}}$ for the $A$ state of $\mathrm{CN}, 9235 \mathrm{~cm}^{-1}$, is considerably higher than the term value for the $A$ state in $\operatorname{SiN}, 2032 \mathrm{~cm}^{-1}$. A simple molecular orbital picture might lead one to expect a decrease in bond order from 3 to 2.5 upon photodetachment from either $\mathrm{CN}^{-}$or $\mathrm{SiN}^{-}$accompanied by an increased $r_{\mathrm{e}}$. In fact, the bond length for detachment to the $\mathrm{X}^{2} \Sigma^{+}$state decreases in both cases: $\Delta r_{\mathrm{e}}=-0.005 \AA$ for $\mathrm{CN}^{-}$and $-0.032 \AA$ for $\mathrm{SiN}^{-}$. In $\mathrm{CN}^{-}$, the very small bond length change was attributed to the nonbonding character of the $\sigma$ highest occupied molecular orbital (HOMO) from which detachment occurs to form the neutral $X$ ${ }^{2} \Sigma^{+}$state. ${ }^{8}$ It thus appears that the $7 \sigma \mathrm{HOMO}$ in $\mathrm{SiN}^{-}$has some antibonding character.

\section{Conclusions}

The anion photoelectron spectra of $\mathrm{SiN}^{-}$have been recorded at 355 and $266 \mathrm{~nm}$ photodetachment wavelengths. We obtained the first experimental spectroscopic parameters for the anion 
ground state, $r_{\mathrm{e}}=1.604 \pm 0.005 \AA$ and $\omega_{\mathrm{e}}=1130 \pm 20 \mathrm{~cm}^{-1}$. We determined the adiabatic electron affinity of $\mathrm{SiN}$ as 2.949 $\pm 0.008 \mathrm{eV}$ and the $\mathrm{SiN}^{-}$dissociation energy as $6.24 \pm 0.14$ $\mathrm{eV}$. The PE spectra also confirm the most recent literature values of the term value for the $\mathrm{SiN} \mathrm{A}{ }^{2} \Pi$ state, $2032 \mathrm{~cm}^{-1}$. Finally, we tentatively assigned a small spectral feature to the transition between the anion first excited electronic state $\left(a^{3} \Sigma^{+}\right)$and the $b^{4} \Pi$ state of $\mathrm{SiN}$.

Acknowledgment. We gratefully acknowledge the support from the National Science Foundation under Grant No. DMR0139064 .

\section{References and Notes}

(1) Katz, R. N. Science 1980, 208, 841.

(2) Dusza, J.; Sajgalik, P.; Bastl, Z.; Kavecansky, V.; Durisin, J. J Mater. Sci. Lett. 1992, 11, 208.

(3) Schmidt, H.; Nabert, G.; Ziegler, G.; Goretzki, H. J. Eur. Ceram. Soc. 1995, 15, 667.

(4) Amaral, M.; Oliveira, F. J.; Belmonte, M.; Fernandes, A. J. S.; Costa, F. M.; Silva, R. F. Surf. Eng. 2003, 19, 410.

(5) Tarasov, I.; Dybiec, M.; Ostapenko, S.; Torchynska, T. V. Physica B: Condens. Matter 2003, 340, 1124.

(6) Paszkowicz, W.; Minikayev, R.; Piszora, P.; Knapp, M.; Bahtz, C.; Recio, J. M.; Marques, M.; Mori-Sanchez, P.; Gerward, L.; Jiang, J. Z Phys. Rev. B 2004, 69.

(7) Zou, L. H.; Park, D. S.; Cho, B. U.; Huang, Y.; Kim, H. D. Mater. Lett. 2004, 58, 1587.

(8) Bradforth, S. E.; Kim, E. H.; Arnold, D. W.; Neumark, D. M. J. Chem. Phys. 1993, 98, 800

(9) Asmis, K. R.; Taylor, T. R.; Neumark, D. M. Chem. Phys. Lett. 1998, 295, 75 .

(10) Gomez, H.; Taylor, T. R.; Zhao, Y.; Neumark, D. M. J. Chem. Phys. 2002, 117, 8644 .
(11) Jevons, W. Proc. R. Soc. London 1913, 89A, 187

(12) Mulliken, R. S. Phys. Rev. 1925, 26, 319

(13) Bredohl, H.; Dubois, I.; Houbrechts, Y.; Singh, M. Can. J. Phys 1976, 54, 680

(14) Foster, S. C. J. Mol. Spectrosc. 1984, 106, 369.

(15) Yamada, C.; Hirota, E. J. Chem. Phys. 1985, 82, 2547.

(16) Yamada, C.; Hirota, E.; Yamamoto, S.; Saito, S. J. Chem. Phys. 1988, $88,46$.

(17) Foster, S. C. J. Mol. Spectrosc. 1989, 137, 430

(18) Saito, S.; Endo, Y.; Hirota, E. J. Chem. Phys. 1983, 78, 6447.

(19) Naulin, C.; Costes, M.; Moudden, Z.; Ghanem, N.; Dorthe, G. Chem. Phys. Lett. 1993, 202, 452.

(20) Curtiss, L. A.; Raghavachari, K.; Trucks, G. W.; Pople, J. A. J. Chem. Phys. 1991, 94, 7221.

(21) Peterson, K. A.; Woods, R. C. J. Chem. Phys. 1989, 90, 7239.

(22) Cai, Z. L.; Martin, J. M. L.; Francois, J. P.; Gijbels, R. Chem. Phys. Lett. 1996, 252, 398

(23) Cai, Z. L.; Martin, J. M. L.; Francois, J. P. J. Mol. Spectrosc. 1998, $188,27$.

(24) Singh, P. D.; Sanzovo, G. C.; Borin, A. C.; Ornellas, F. R. Mon. Not. R. Astron. Soc. 1999, 303, 235.

(25) Kalcher, J. Phys. Chem. Chem. Phys. 2002, 4, 3311.

(26) Midda, S.; Das, A. K. J. Mol. Struct.: THEOCHEM 2003, 640, 183.

(27) Midda, S.; Das, A. K. Eur. Phys. J. D 2003, 27, 109

(28) Metz, R. B.; Weaver, A.; Bradforth, S. E.; Kitsopoulos, T. N.; Neumark, D. M. J. Phys. Chem. 1990, 94, 1377.

(29) Xu, C. S.; Burton, G. R.; Taylor, T. R.; Neumark, D. M. J. Chem. Phys. 1997, 107, 3428

(30) Cooper, J.; Zare, R. N. J. Chem. Phys. 1968, 48, 942.

(31) Arnold, D. W.; Bradforth, S. E.; Kitsopoulos, T. N.; Neumark, D. M. J. Chem. Phys. 1991, 95, 8753.

(32) Blondel, C.; Delsart, C.; Goldfarb, F. J. Phys. B: At. Mol. Opt. Phys. 2001, 34, 2757.

(33) Huber, K. P.; Herzberg, G. Molecular Spectra and Molecular Structure. IV. Constants of Diatomic Molecules; Van Nostrand Reinhold: New York, 1979 